Engineering Center and Office of Naval Research at Maryland, by the state of Texas through the Texas Center for Superconductivity at the University of Houston, and the Department of Energy at Argonne.

Correspondence and requests for materials should be addressed to T. V. (e-mail: venky@squid.umd.edu).

\section{Complete photonic bandgaps in 12-fold symmetric quasicrystals}

\author{
M. E. Zoorob, M. D. B. Charlton*, G. J. Parker ${ }^{\star}$, J. J. Baumberg ${ }^{\star} \dagger$ \\ \& M. C. Netti $\dagger$
}

${ }^{*}$ Department of Electronics and Computer Science, $\dagger$ Department of Physics and Astronomy, University of Southampton, Southampton S017 1BJ, UK

Photonic crystals are attracting current interest for a variety of reasons, such as their ability to inhibit the spontaneous emission of light ${ }^{1,2}$. This and related properties arise from the formation of photonic bandgaps, whereby multiple scattering of photons by lattices of periodically varying refractive indices acts to prevent the propagation of electromagnetic waves having certain wavelengths. One route to forming photonic crystals is to etch twodimensional periodic lattices of vertical air holes into dielectric slab waveguides ${ }^{3-7}$. Such structures can show complete photonic bandgaps ${ }^{8-10}$, but only for large-diameter air holes in materials of high refractive index (such as gallium arsenide, $n=3.69$ ), which unfortunately leads to significantly reduced optical transmission when combined with optical fibres of low refractive index. It has been suggested that quasicrystalline (rather than periodic) lattices can also possess photonic bandgaps ${ }^{11-14}$. Here we demonstrate this concept experimentally and show that it enables complete photonic bandgaps - non-directional and for any polarization-to be realized with small air holes in silicon nitride $(n=2.02)$, and even glass $(n=1.45)$. These properties make photonic quasicrystals promising for application in a range of optical devices ${ }^{14-18}$.

On the basis of previous theoretical studies ${ }^{3,9,10,19,20}$, it is evident that the anisotropy of a photonic bandgap (PBG) is dependent on the symmetry of the photonic crystal lattice. As the order of the symmetry increases, the Brillouin zone becomes more circular, resulting in a complete bandgap. The highest level of symmetry found in periodic lattices is six; however, much higher levels of symmetry can be achieved using the more complex geometries of quasicrystals. These quasicrystal photonic lattices eliminate waveguiding modes coincident with the PBG. We investigated photonic quasicrystals based on a random square-triangle tiling system ${ }^{21,22}$, which possess 12 -fold symmetry. These were modelled using a finite difference time domain (FDTD) method to optimally design silicon nitride waveguide structures producing PBGs in the visible/nearinfrared spectral region.

The motivation for using a quasicrystal is to maintain the periodic scattering of light while reducing the orientational order of the system so that it is more isotropic. This is achieved using a random ensemble of squares and equilateral triangles to form the lattice of air pores, which are separated by a distance $a$. In order to exhibit long-range 12-fold symmetry, a large number of cells are required. We used the Stampfli inflation rule, with dodecahedral parent cells (Fig. 1a, dashed lines) generating the offspring dodecagons (Fig. 1a, solid lines). The resulting arrangement of air rods in the waveguide dielectric layers is generated by placing holes at the vertices as shown in Fig. 1b (plan view) and Fig. 1c (section). The photonic quasicrystals examined are composed of 150-nm-diameter air rods arranged on a pitch $a$ of $260 \mathrm{~nm}$. The 260 -nm-thick waveguide of silicon nitride is capped above and below by silicon dioxide to confine the light in the waveguide (Fig. 1c).

Clues to the scattering properties of the quasicrystal may be found in the reciprocal lattice space (Fig. 1d). The large parent cell generates the dodecahedral 'Brillouin zone' indicated, while the edges of Fig. 1d extend to the basic reciprocal vector $2 \pi / a$. A triangular or square lattice of the same nearest-neighbour distance would have a sparse reciprocal lattice with points at the corners; however, the quasicrystal has many additional symmetries that average out the anisotropy, but provide additional resonant scattering conditions.

We model the propagation through 28 rows of the structure using an FDTD method. This directly tracks the scattering of waves from a gaussian wavepacket entering the quasicrystal, and is based on a centred difference spatial step ${ }^{23}$ to reduce transport errors from scattering in both forward and backward directions. Figure 2a shows the predicted bandgap limits as a function of the air filling fraction $\beta$, for a pitch $a=260 \mathrm{~nm}$, and TE-polarized light propagating along the $\Gamma$-J direction. We note that even for small values of $\beta$, a bandgap is apparent. At $\beta=10 \%$, the ratio of the bandgap to the central frequency $\left(\Delta \omega / \omega_{0}\right)$ is $13 \%$, whereas for a triangular lattice with the same filling fraction and geometry $\Delta \omega / \omega_{0}=2.5 \%$ in the same direction.
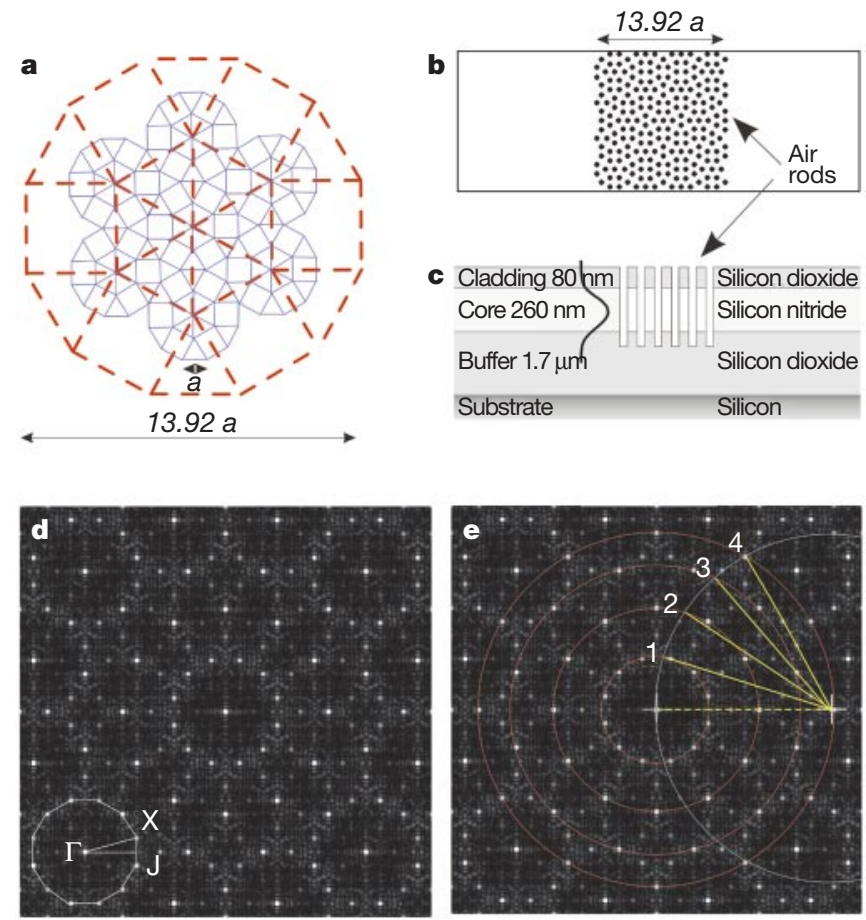

Figure 1 Scheme of the basic quasicrystal elementary unit and its implementation in a planar waveguide. a, Quasicrystal pattern based on square-triangular tiling, grown using random Stampfli inflation from parent lattice (dashed red lines) to produce offspring tiling (solid lines). $a$ is the distance between the air rods (pitch) and $13.92 a\left(=a(2+\sqrt{3})^{2}\right)$ is the total size of the parent lattice. $\mathbf{b}$, Plan of air rods placed at quasicrystal vertices and etched though the planar waveguide. c, Cross-section of waveguide structure, consisting of 260 -nm-thick silicon nitride $(n=2.04)$ sandwiched between two silicon dioxide $(n=1.46)$ layers grown on a thick silicon buffer layer. The depth of holes and calculated waveguide mode in planar regions are also shown. d, Representation of the quasicrystal reciprocal lattice, showing 12-fold symmetry. The Brillouin zone (white dodecagon) corresponds to the supercell shown dashed in a. e, Ewald sphere construction for the 633-nm light used in the experiment. $k_{\text {in }}$ (dashed) defines the radius of the sphere (grey) whose intersection with planes of air rods (red circles in reciprocal space) gives the diffraction directions $k_{\text {in }}$ (marked 1-4). 
Transmission spectra were calculated in all directions using the FDTD method for a photonic quasicrystal with $a=260 \mathrm{~nm}$ and $\beta=$ $28 \%$. Figure $2 \mathrm{~b}$ shows the predicted transmission for both TE- and TM-polarized modes along the $\Gamma$-J direction. We use the normalized frequencies $a / \lambda$ for conveniently comparing different structures. The complete PBG persists from $0.158 a / \lambda$ to $0.190 a / \lambda$ in all directions for the first bandgap. A higher-order bandgap is also seen from $0.408 a / \lambda$ to $0.418 a / \lambda$. The normalized width of the primary bandgap is $\Delta \omega / \omega_{0}=33.0 \%$ for TE modes, and $\Delta \omega / \omega_{0}=20.5 \%$ for TM modes. In contrast to regular lattices, the TM PBG lies exactly in the middle of the TE $\mathrm{PBG}^{24}$. This gives rise to a $65.5 \%$ overlap in the complete PBG for the two different polarization states, compared to the total absence of a complete PBG for triangular and hexagonal lattice structures with the same air filling fraction ${ }^{3}$. In the longwavelength limit $(a / \lambda<0.10)$, the transmission reaches unity. At these wavelengths the fine structure is not resolved by the propagating waves, and the quasicrystal is seen as homogeneous material of reduced refractive index.

The quasicrystal bandgaps occur at lower frequencies than in the

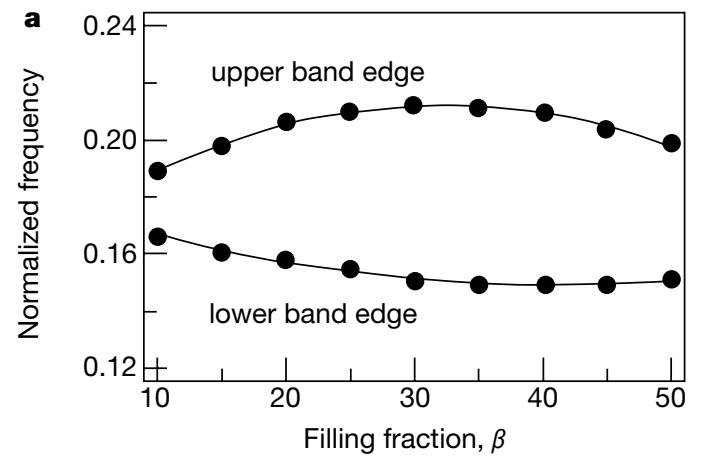

b
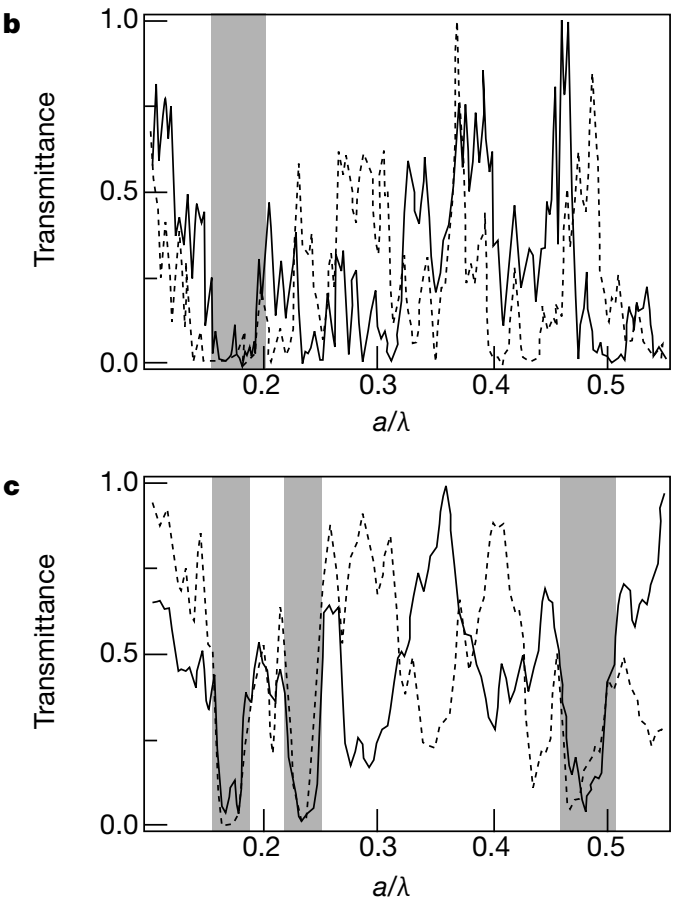

Figure 2 Theoretical calculation. a, Photonic bandgap extrema as a function of filling fraction, $\beta$, for $a=260 \mathrm{~nm}$ with light along $\Gamma-J$. The gaps vary by less than $2 \%$ in different directions. $\mathbf{b}$, Transmission spectra for TE (dashed line) and TM (solid line) polarizations as a function of normalized frequencies $a / \lambda(a=260 \mathrm{~nm})$, calculated for a quasicrystal in SiN waveguide having air-filling fraction $\beta=28 \%$, and $\mathbf{c}$, for the same lattice in glass with $\beta=30 \%$. The shaded areas mark the main gaps and their superposition for both TE and TM modes. triangular and hexagonal crystals. The normalized midgap frequencies for TE and TM are $0.170 a / \lambda$ and $0.176 a / \lambda$, respectively, compared to $\sim 0.375 a / \lambda$ for regular lattices ${ }^{24}$. In contrast to regular lattice structures, nearest-neighbour lattice vectors no longer define the 'Brillouin zone'. As a consequence of quasicrystal symmetry, the 'Brillouin zone' is instead defined by lattice vectors joining air rods several periods away, as seen by the 'Brillouin zone' in Fig. 1d. Further simulations show that the photonic bandgap remains open for very low index materials such as glass $(n=1.45)$ with an air filling fraction $\beta$ of only $30 \%$. The calculated transmission shown in Fig. 2c confirms that it is should be possible in practice to integrate photonic quasicrystal devices with optical fibre systems.

Quasicrystal waveguides have been fabricated with a range of parameters, and demonstrated good reproducibility and stability. Figure 3a shows an scanning electron micrograph of a typical device from which the pitch of $260 \mathrm{~nm}$ and a fill fraction of $28 \%$ are measured. To confirm the enhanced diffraction predicted from the quasicrystal reciprocal lattice, the far-field in-plane diffraction is shown in Fig. 3b. TE-polarized light at a wavelength of $633 \mathrm{~nm}$ enters from a cleaved waveguide face on the lower edge, and propagates up to the horizontal line of the photonic quasicrystal. Many diffracted beams are observed, which can be compared to simple theory using the Ewald sphere construction in Fig. 1e. The Bragg scattering condition $\mathbf{k}_{\text {out }}=\mathbf{k}_{\text {in }}+\mathbf{G}$, where $\mathbf{G}$ is a reciprocal lattice vector, can be represented by a circle centred at $\mathbf{k}_{\mathrm{in}}=2 \pi / \lambda_{\mathrm{in}}$, which indicates photon scattering where it intersects the reciprocal lattice points. Note that it is necessary to take into account the
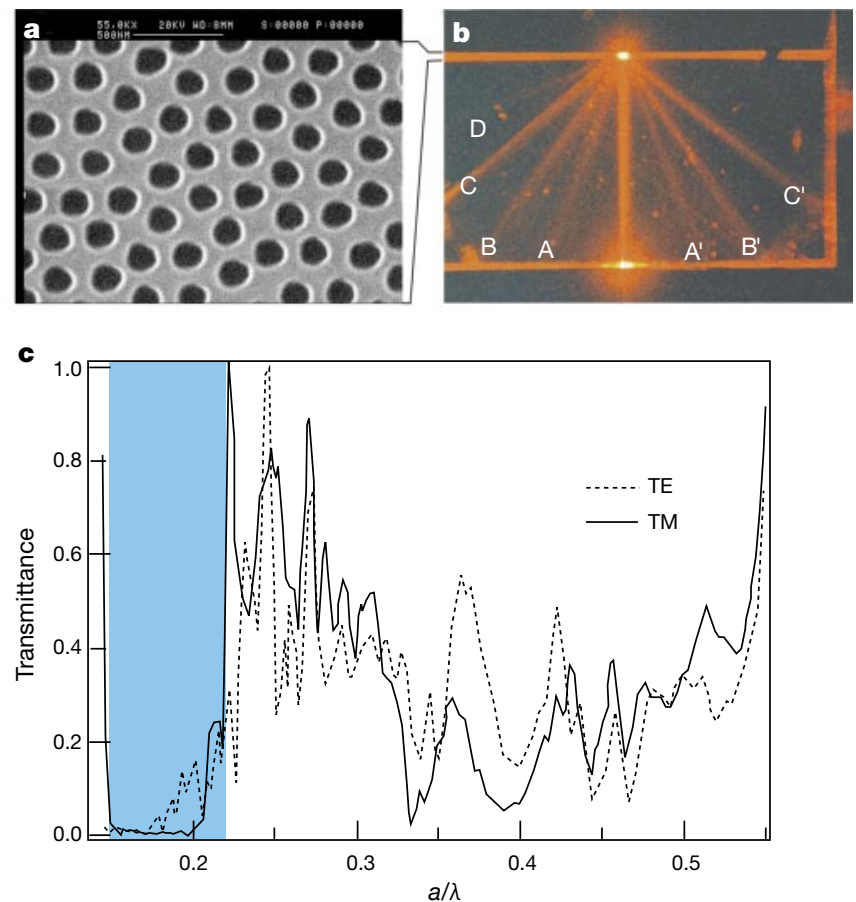

Figure 3 Experimental results. a, SEM image of a 12-fold symmetric photonic quasicrystal structure. $\mathbf{b}$, Photograph demonstrating the multiple beam diffracted reflections at the entrance face of the photonic quasicrystal. The illuminating laser $\lambda=$ $633 \mathrm{~nm}$, compared to the photonic quasicrystal pitch of $a=260 \mathrm{~nm}$ and a filling fraction of $\beta=0.28$. The angles of the diffracted beams $A^{\prime}-C^{\prime}$ and $A-D$ correspond to the $1-4$ diffraction directions calculated by means of the Ewald sphere (Fig. 1e). The angle values are reported in Table 1. c. Experimental transmission in a 12-fold photonic quasicrystal with $a=260 \mathrm{~nm}, \beta=28 \%$ along $\Gamma-J$. The laser system used in these measurements allowed us to cover a spectrum from 450 to $1,800 \mathrm{~nm}$. The curves are corrected to account for short-wavelength scattering from the waveguide using the normalization functions $\exp \left\{-(850 \mathrm{~nm} / \lambda)^{3}\right\}$ for TE and $\exp \left\{-(900 \mathrm{~nm} / \lambda)^{2.5}\right\}$ for TM. The blue area highlights the gaps for both TE (dashed line) and TM (solid line) polarization. 
effective refractive index of the etched area, which affects the size of the Ewald sphere and hence the $\mathbf{k}_{\text {out }}$ direction. This orientationdependent effective index is an approximation, which is only valid in the near-isotropic quasicrystals. The comparison between observed and predicted angles of diffraction is reported in Table 1, and it shows reasonable agreement. Thus, the scattered wavevectors clearly demonstrate the 12 -fold symmetry produced by the quasicrystal nanostructures and confirms their successful fabrication.

Transmission measurements were performed by spectrally resolving the light propagating through the lattice in different directions. The spectra reported in Fig. 3c show (we believe for the first time) experimental evidence for complete bandgaps in quasicrystals for both TE and TM polarizations. The experiments used a white light continuum produced by focusing pulses $(1 \mu \mathrm{J}$, $100 \mathrm{fs}$ ) from a regenerative amplifier tuned to $850 \mathrm{~nm}$ into $1 \mathrm{~mm}$ of sapphire. The availability of such high-brightness ultra-broadband laser sources facilitates high-accuracy transmittance measurements from 450 to $1,800 \mathrm{~nm}$ through the waveguide. The use of achromatic optics and a carefully designed optical fibre spatial filter provided excellent collimation and pointing properties for coupling into the planar waveguide. This special optical fibre also provided extra continuum generation in the range 0.8 to $1.8 \mu \mathrm{m}$ for measurements in the near-infrared. Because the air holes also cause scattering losses, the spectra in Fig. 3c have been normalized to account approximately for the decreased throughput at shorter wavelengths.

The main features of the transmission spectra in Fig. $3 \mathrm{c}$ are the presence of sharp gaps amid strongly modulated structure, very similar to that predicted by the simulations. This differs dramati-
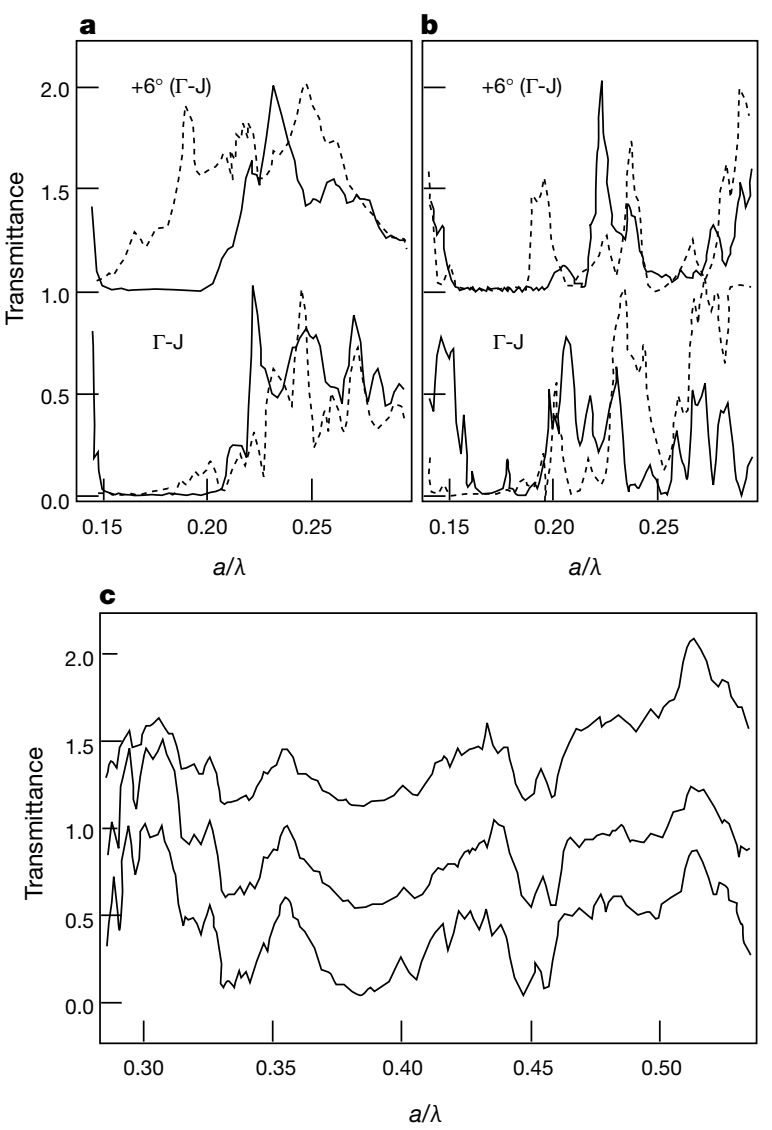

Figure 4 Angular dependence of the primary PBG and reproducibility of transmittance. a, Experimental transmission along the $\Gamma-J$ direction (lower pair of curves) and $6^{\circ}$ between $\Gamma-J$ and $\Gamma-X$ directions (upper pair of curves) for both TE (dashed lines) and TM (solid lines) modes. $\mathbf{b}$, Theoretical transmission for the same angles and frequency range as in a. c. Scanning of transmission at different points ( 1 -mm spacing) along the device.

\begin{tabular}{|c|c|c|c|}
\hline Experimental beam & Measured angle & Theoretical beam $†$ & Theoretical angle \\
\hline $\mathrm{A}, \mathrm{A}^{\prime}$ & $20.9,18.8$ & 1 & 18.0 \\
\hline$B, B^{\prime}$ & $36.3,33.0$ & 2 & 34.6 \\
\hline $\mathrm{C}, \mathrm{C}^{\prime}$ & $51.3,50.1$ & 3 & 51.3 \\
\hline $\mathrm{D}$ & 62.0 & 4 & 62.5 \\
\hline
\end{tabular}

Shown are experimental and theoretical angles of diffracted reflections from a photonic quasicrystal with $a=260 \mathrm{~nm}$ and $\lambda=633 \mathrm{~nm}$.

${ }^{\star}$ Fig. 3b.

†Fig. 1e.

cally from regular lattice structures, which exhibit less spectral structure in keeping with their sparser reciprocal lattices ${ }^{24}$. The primary bandgap for both TE and TM modes are exactly centred at $0.17 a / \lambda$, showing an extinction ratio as good as $10^{-3}$, in excellent agreement with the theory. In addition, the experimental transmittances show higher-order bandgaps for both TE and TM polarized gaps centred at $0.352 a / \lambda$ and $0.413 a / \lambda$ with an extinction ratio of about $90 \%$.

The experimental investigation of the angular dependence of the main PBG at $0.17 a / \lambda$ is shown in Fig. 4a. The lower and upper transmission spectra were obtained at normal incidence and $6^{\circ}$ around the $\Gamma$-J direction, respectively. The limit of the sensitivity of the infrared detector is at about $0.14 a / \lambda$. The theoretical simulations along the same directions are given in Fig. 4b, showing a very good agreement with the experimental spectra.

Reproducibility and fabrication tolerances were also investigated by sampling the transmission characteristics along the photonic quasicrystal device at several different positions (spaced by $\sim 1 \mathrm{~mm}$ ) as shown in Fig. 4c. The fine spectral details of all the different transmission spectra are maintained, indicating excellent fabrication quality as confirmed by Fig. 3a.

All experimental spectra show the same fine structure as that calculated in Fig. 2b, and in particular the predicted angular and polarization dependence. Although the primary PBG width is reduced by a factor of 1.05 compared to theory, the central frequency is identical. This small mismatch is not surprising if one takes into account that the FDTD calculation is a two-dimensional model that neglects the real three-dimensional character of waveguiding in a planar structure. This discrepancy between the theoretical and experimental results clearly demonstrates that although the refractive index periodicity has a two-dimensional translational symmetry, a full three-dimensional treatment needs to be pursued for these structures. This is the case even for regular photonic crystals, where recent experimental and theoretical results show similar discrepancies in transmission spectra ${ }^{5,24-26}$. Full threedimensional theoretical modelling of these waveguiding quasicrystal devices is a non-trivial task, given the lack of long range periodicity and the added complexity of satisfying waveguiding conditions. Promising results are predicted on the basis of previous initial investigations on the three-dimensional modelling of periodic structures $^{27}$. This work is currently in progress, and as shown here, an appropriate assembly of air cylinders in a low-refractiveindex-dielectric waveguide is a promising technology for controlling the flow of light.

Received 16 December 1999; accepted 28 February 2000

Yablonovitch, E. Inhibited spontaneous emission in solid-state physics and electronics. Phys. Rev. Lett. 58, 2059-2062 (1987).

2. Joannopoulos, J. D., Meade, R. D. \& Winn, J. N. Photonic Crystals (Princeton Univ. Press, New York, 1995).

3. Charlton, M. D. B. \& Parker, G. J. Guided mode analysis, and fabrication of a 2-dimensional visible photonic band structure confined within a planar semiconductor waveguide. Mater. Sci. Eng. B 49, 155-165 (1997).

4. Joannopoulos, J. D., Villeneuve, P. R. \& Fan, S. Photonic crystals: putting a new twist on light. Nature 386, 143-149 (1997).

5. Gadot, F. et al. Experimental demonstration of complete photonic bandgap in graphite structure. Appl. Phys. Lett 71, 1780-1782 (1997).

6. Krauss, T. F., De La Rue, R. M. \& Brand, S. Two-dimensional photonic-bandgap structures operating at near-infrared wavelengths. Nature 383, 699-702 (1996).

7. Atkin, D. N., Russell, P. S. J., Birks, T. A. \& Roberts, P. J. Photonic band structure of guided Bloch 
modes in high index films fully etched through with periodic microstructure. J. Mod. Opt 43, 10351053 (1996)

8. Plihal, M. \& Maradudin, A. A. Photonic band-structure of 2-dimensional systems-the triangular lattice. Phys. Rev. B 44, 8565-8571 (1991).

9. Cassagne, D., Jouanin, C. \& Bertho, D. Hexagonal photonic-band-gap structures. Phys. Rev. B 53, 7134-7142 (1996)

10. Barra, A., Cassagne, D. \& Jouanin, C. Existence of two-dimensional absolute photonic band gaps in the visible. Appl. Phy. Lett. 72, 627-629 (1998).

11. Chan, Y. S., Chan, C. T. \& Liu, Z. Y. Photonic band gaps in two dimensional photonic quasicrystals. Phys. Rev. Lett. 80, 956-959 (1998).

12. Cheng, S. S. M., Li, L., Chan, C. T. \& Zhang, Z. Q. Defect and transmission properties of twodimensional quasiperiodic photonic band-gap systems. Phys. Rev. B 59, 4091-4099 (1999).

13. Krauss, T. F. \& De la Rue, R. M. Photonic crystals in the optical regime-past, present and future. Prog. Quant. Electron. 23, 51-96 (1999).

14. Charlton, M. D. B., Parker, G. J. \& Zoorob, M. E. Recent developments in the design and fabrication of visible photonic band gap waveguide devices. J. Mater. Sci. 10 (Materials in Electronics), 429-440 (1999).

15. Foresi, J. S. et al. Photonic-bandgap microcavities in optical waveguides. Nature 390, 143-145 (1997)

16. Temelkuran, B. \& Ozbay, E. Experimental demonstration of photonic crystal based waveguides. Appl. Phys. Lett 74, 486-488 (1999).

17. Kosada, H. et al. Superprism phenomena in photonic crystals. Phys. Rev. B 58, R10096-R10099 (1998).

18. Ohetera, Y., Sato, T., Kawashima, T., Tamamura, T. \& Kawakami, S. Photonic crystal polarisation splitters. Electron. Lett. 35, 1271-1272 (1999).

19. McGurn, A. R. \& Maradudin, A. A. Weak transverse localisation of light scattered incoherently from a one-dimensional random metal-surface. J. Opt. Soc. Am B 10, 539-545 (1993).

20. Anderson, C. M. \& Giapis, K. P. Symmetry reduction in group $4 \mathrm{~mm}$ photonic crystals. Phys. Rev. B 56, $7313-7320$ (1997)

21. Zoorob, M. E., Charlton, M. D. B. \& Parker, G. J. Proc. Inst. Phys. PREP 99 161-164 (1999).

22. Oxborrow, M., Henley, C. L. Random square-triangle tilings: A model for twelvefold-symmetric quasicrystals. Phys. Rev. B 48, 6966-6998 (1993)

23. Yee, K. S. Numerical solutions of initial boundary value problems involving Maxwell's equation in isotropic media. IEEE Trans. Antennas Propagat. AP-14, 302-307 (1966).

24. Netti, M. C., Charlton, M. D. B., Parker, G. J. \& Baumberg, J. J. Visible photonic bandgap engineering in silicon nitride waveguides. Appl. Phys. Lett. (in the press).

25. Feng, X. -P. \& Arakawa, Y. Off-pane angle dependence of photonic band gap in a two-dimensional photonic crystal. IEEE. J. Quantum. Electron. 32, 535-542 (1996).

26. Labilloy, D. et al. Quantitative measurement of transmission, reflection, and diffraction of twodimensional photonic band gap structures at near infrared wavelengths. Phys. Rev. Lett 79, 4147-4150 (1997).

27. Ho, K. M., Chan, C. T., Soukoulis, C. M., Biswas, R. \& Sigalis, M. Photonic band gaps in three dimensions: new layer-by-layer periodic structures. Solid State Commun. 89, 413-416 (1994).

\section{Acknowledgements}

We thank the holey-fibre group of the Southampton Optoelectronics Research Centre for supplying samples. This work was supported by the EPSRC, the HEFCE and the University of Southampton.

Correspondence and requests for materials should be addressed to G.J.P. (e-mail: gjp@ecs.soton.ac.uk). tures-atoms that are sufficiently well bound to a surface to be stable at higher temperatures require a stronger tip interaction to be moved. This situation differs significantly from the idealized weakly interacting tips ${ }^{12,13}$ of scanning tunnelling or atomic force microscopes. Here we demonstrate that precise positioning of atoms on a copper surface is possible at room temperature. The triggering mechanism for the atomic motion unexpectedly depends on the tunnelling current density, rather than the electric field or proximity of tip and surface.

For $\mathrm{Br}$ adsorbed on $\mathrm{Cu}(001)$, we find that whereas normal stable and atom-resolved scanning tunnelling microscope (STM) images can be obtained at lower tunnel currents, the adsorbed atoms start moving if the images are taken with a tunnel current above a few nanoamps. The tunnel current provides a remarkably well defined motion-control parameter. The STM images reported here were taken at room temperature with an Omicron ultrahigh vacuum (UHV) STM. The line scans are horizontal, and start at the bottom of the image. The $\mathrm{Cu}(001)$ was cleaned by standard UHV procedures and the bromine was then deposited at low coverages using an in situ electrochemical cell doser ${ }^{14}$.

Figure 1 shows an image where the tunnel current is changed from 3 to $1 \mathrm{nA}$ halfway up the scan. In the first, lower part of the image ( $3 \mathrm{nA})$, most of the $\mathrm{Br}$ adsorbates appear as a series of tracks, perpendicular to the line scan. In the upper $(1 \mathrm{nA})$ part of the image, the $\mathrm{Br}$ atoms are in fixed, well defined locations on the surface. The tracks in the lower part of the image are in fact single atoms being driven in front of the tip, and hopping from one $\mathrm{Cu}$ surface site to another. Figure 2 is the same surface region just afterwards, but with a current of $1 \mathrm{nA}$, which is below the threshold to induce motion. The atoms marked ABCD are seen to be still in the same place as they were left in Fig. 1, but the area below them has been 'swept' clear of most $\mathrm{Br}$ adsorbates. For $95 \%$ of our images, the underlying metal lattice has a corrugation too small to observe, as is expected for low-index metal surfaces ${ }^{15}$; but occasionally a tip structure occurs which enables the metal atoms to be seen ${ }^{15}$. We find that the tip structure makes no significant difference to the general character of the Br motion we observe. Therefore, in the remaining figures we show these rather rarer types of image where the metal lattice can be seen, to make the local nature of the atom motion completely clear.

The image shown in Fig. 3, taken at $2 \mathrm{nA}$, clearly shows the underlying $\mathrm{Cu}(001)$ lattice registry. Most atoms are hopping

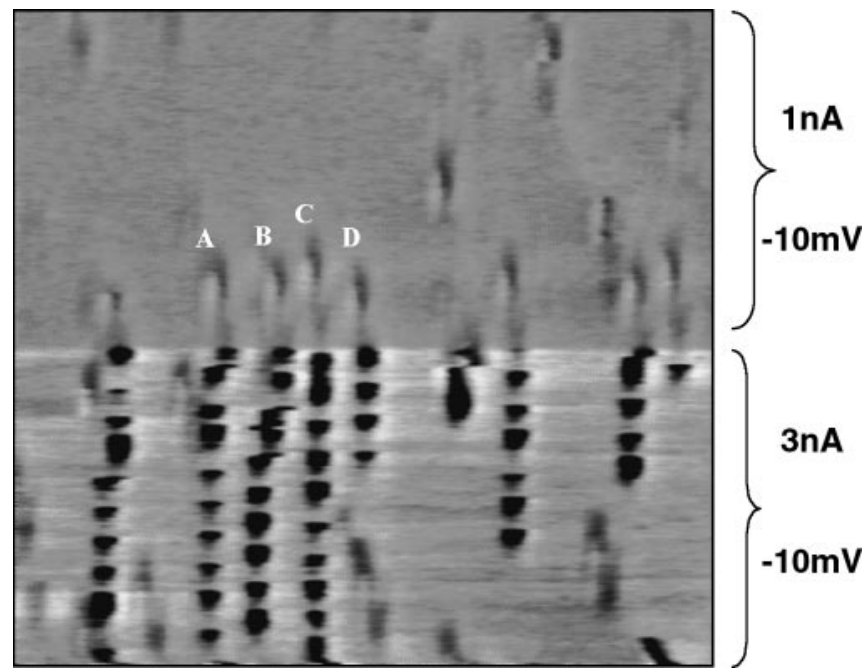

Figure 1 STM image, $60 \AA \times 60 \AA$, of $\mathrm{Br}$ on $\mathrm{Cu}(100)$. The tunnel current is reduced halfway up the image. The tracks in the 3-nA (lower half) area are single atoms hopping from site to site. When the current is reduced the bromine atoms stop moving, for example those marked $A, B, C$ and $D$. 